\title{
A new insight into the treatment of renal anemia with HIF stabilizer
}

\author{
Satoru Kuriyama ${ }^{1,2^{*}}$, Yukio Maruyama ${ }^{3}$ and Hirokazu Honda ${ }^{4}$
}

\begin{abstract}
The long-term clinical experiences with recombinant human erythropoietin (rHuEPO) and its analog derivatives have clearly proven that correction of anemia with erythropoiesis stimulating agent (ESA) not only reduces blood transfusion and improves patients' QOL but has multiple benefits for the concurrent complications of CKD such as Cardio-Renal-Anemia (CRA) syndrome and/or malnutrition-inflammation-atherosclerosis (MIA) syndrome. Unlike ESA, the newly available agent, hypoxia-inducible factor (HIF) stabilizer, stimulates endogenous erythropoietin (EPO) by mimicking hypoxia with HIF prolyl hydroxylase domain enzyme (HIF-PHD) inhibition. The phase 2 and 3 clinical studies have shown that HIF stabilizers are as efficacious as ESA in ameliorating renal anemia. Whether the same clinical benefits on CRA and MIA syndrome hold true in patients given HIF stabilizers is a matter for future debate. Given that HIF stabilizers act on the multiple target genes, the use of this novel agent may lead to unwanted adverse events.

Launching HIF stabilizers into the treatment of renal anemia provokes a concern about how this alternative treatment will be taken up in the daily clinical practice. However, guideline-oriented strategies on how to use HIF stabilizer is not available at this limited point due to scant clinical information. Nevertheless, this opinion-based review provides a future insight into the management of renal anemia with HIF stabilizer by reference to the past experiences with ESA. HIF stabilizers can preferably be indicated for CRA syndrome at pre-dialysis stage, ESA resistant anemia at advanced CKD stage, and perhaps for dysregulated iron metabolism akin to MIA syndrome in patients on dialysis.
\end{abstract}

Keywords: Hypoxia inducible factor (HIF) stabilizer, Hypoxia, Erythropoiesis stimulating agent (ESA), CRA syndrome, MIA syndrome, Iron metabolism

\section{Introduction}

The epoch-making recombinant human erythropoietin (rHuEPO) came into clinical practice for renal anemia in chronic kidney disease (CKD) patients on dialysis in 1990, and its indication was extended to those at predialysis stage in 1994 in Japan. The pharmacological potency of correction of anemia with rHuEPO and its pharmacological analogues was so efficacious that most

\footnotetext{
* Correspondence: kuriyamas218@yahoo.co.jp

${ }^{1}$ Jikei University School of Medicine, 3-25-8, Nishi-shinbashi, Minato-ku, Tokyo 105-8471, Japan

${ }^{2}$ Nephrology \& Hypertension Research Unit, Internal Medicine, Miho Clinic, Shin-Osaki-kangyo Bld 2F, Osaki, Shinagawa-ku, Tokyo 141-0032, Japan Full list of author information is available at the end of the article
}

of the CKD patients benefited from the treatment. One of the remarkable effects of the erythropoiesis stimulating agents (ESA) was the reduction in blood transfusion that contributed to the prevention of blood-transfusion-borne infections such as viral hepatitis and other intractable lifethreatening diseases before the Era of ESA. ESA has proven to be cardio-renal protective, reducing cardio-renal-anemia (CRA) syndrome, a highly risky cardiovascular (CV) condition associated with CKD. Indeed, a large body of evidence has clearly proven that ESA regresses left ventricular hypertrophy (LVH) and retards the progression of CKD $[1,2]$. In addition, ESA also improves malnutritioninflammation-atherosclerosis (MIA) syndrome, an abnormal

(c) The Author(s). 2020 Open Access This article is licensed under a Creative Commons Attribution 4.0 International License, which permits use, sharing, adaptation, distribution and reproduction in any medium or format, as long as you give appropriate credit to the original author(s) and the source, provide a link to the Creative Commons licence, and indicate if changes were made. The images or other third party material in this article are included in the article's Creative Commons licence, unless indicated otherwise in a credit line to the material. If material is not included in the article's Creative Commons licence and your intended use is not permitted by statutory regulation or exceeds the permitted use, you will need to obtain permission directly from the copyright holder. To view a copy of this licence, visit http://creativecommons.org/licenses/by/4.0/ The Creative Commons Public Domain Dedication waiver (http://creativecommons.org/publicdomain/zero/1.0/) applies to the data made available in this article, unless otherwise stated in a credit line to the data. 
iron metabolism characterized by the increased hepcidin and serum ferritin levels $[3,4]$.

Lagging about 3 decades behind ESA in 2019, the hypoxia inducible factor (HIF) stabilizer which stimulates endogenous erythropoietin (EPO) by mimicking hypoxia with HIF prolyl hydroxylase domain enzyme (HIF-PHD) inhibition was launched for the first time into the clinical practice of renal anemia in Japan. Its clinical indication was limited only to patients on dialysis at the beginning in 2019, and it was extended to those in predialysis stage in August 2020. Although the pharmacological mode of action of HIF stabilizer to stimulate erythropoiesis is totally different from ESA, the phase-2 and phase- 3 clinical studies have shown that HIF stabilizers are as efficacious as ESA in ameliorating renal anemia [5-19]. However, at this point whether HIF stabilizer exerts the same benefits in patients with CRA and MIA syndrome is a matter for future debate. In addition, upcoming problems to be solved are to what extent the assumptive adverse effects originate from the multiple target genes that the HIF activates will be clinically problematic.

Treatment of renal anemia with HIF stabilizers provokes a clinical concern about how this alternative treatment will be taken up in our daily CKD practice. Clinical guidelines on how to use HIF stabilizer are not yet available due to insufficient clinical information. Therefore, by reference to many clinical experiences with ESA in the past three decades, this opinion-based review may provide anew a future insight into how we clinicians should manage renal anemia with HIF stabilizer.

\section{The differential diagnosis on renal anemia}

The total number of human cells is approximately $30 \times$ $10^{12}$. The largest contributor to the overall number of human cells is red blood cells (RBCs) with a total of 25 $\times 10^{12}$, indicating that $84 \%$ of the total body cells are $\mathrm{RBCs}$ [20]. The regulatory system of RBCs is a dynamic and actively operating one, constantly producing $2.0 \times$ $10^{6}$ (cells/second) and destroying the same number at the same time. Iron is an essential element for erythropoiesis and the regulatory system of iron is basically the closed circuit in which iron in the RBCs is constantly reused, as long as loss of RBCs such as gastrointestinal (GI) bleeding did not happen. Once RBCs are destroyed in the reticuloendothelial system, iron is reused efficiently as heme iron. The total body iron storage is estimated approximately $3 \sim 4 \mathrm{~g}$. In a physiological condition in which there is no blood loss, only $1 \sim 2 \mathrm{mg}$ of iron is excreted from the decidual cells in the GI tracts which is routinely replenished with food.

Causative conditions/diseases for anemia in CKD patients are multifactorial; these include CKD-associated reduced EPO production, loss of blood, iron deficiency, malabsorption of iron, dysregulation of iron metabolism, hemolysis, vitamin B12 and/or folic acid deficiency, use of RAS inhibitors, malnutrition, autoantibodies to EPO receptor, and coincidental hematological disorders such as MDS and aplastic anemia. The definition of renal anemia, in a narrow sense, is a normocytic normochromic anemia without the elevation of reticulocytes. For making a differential diagnosis of renal anemia, measurement of circulating EPO concentration is practically important. In renal anemia in CKD, due to the reduced production of EPO in the kidney, the EPO concentration is normally less than $50 \mathrm{mIU} / \mathrm{mL}$. In contrast, in anemic patients without $C K D$, the most of them have a circulating EPO exceeding $50 \mathrm{mIU} / \mathrm{mL}$. It is also known that patients with acute GI bleeding sometimes have increased EPO concentration above $200 \sim 300 \mathrm{mIU} / \mathrm{mL}$. All in all, the differential diagnostic criteria for renal anemia include; (1) normocytic normochromic anemia without increase in reticulocytes, (2) EPO $<50 \mathrm{mIU} / \mathrm{mL}$, and (3) able to rule out other diseases that cause anemia. Regarding how to make a differential diagnosis on renal anemia, the guidelines published in the Japanese Society of Dialysis and Transplantation (JSDT) described it in detail [21].

\section{Cardio-renal effect of ESA therapy \\ Effect of ESA in patients with CRA syndrome}

Anemia and/or anemia-induced hypoxia increases cardiac burden and accelerates LVH [22]. In general, the more the CKD progresses into the further advanced stage, the more the deterioration of $\mathrm{LVH}$ advances. Patients with extremely advanced LVH could occasionally further deteriorate into congestive heart failure (CHF), which jeopardizes patient's life prognosis. In the presence of renal anemia in CKD patients, the reduced cardiac function further accelerates the decline in renal function, creating a vicious circle so-called the cardio (or cardiovascular)-renal-anemia (CRA) syndrome. CKD increases inflammation and oxidative stress via hypoxia and eventually activates renin-angiotensin-aldosterone system (RAS) via reduced renal perfusion, which also facilitates cardiovascular disease (CVD). CKD also induces sodium and water retention which frequently leads to hypertension. These changes trigger to aggravate LVH and reduce cardia output, which in turn decreases perfusion of the kidney. Inflammation in CVD also becomes a risk for CKD progression. After all, CKD and CVD together with renal anemia create an intractable vicious circle. The concept of CRA syndrome has been widely acknowledged and discussed elsewhere [23] (Fig. 1). Silverberg et al. reported that treatment of anemia with ESA in patients with the CRA syndrome having congestive heart failure (HF) improved not only patients' subjective symptoms, but also the ejection fraction (EF), NYHA classification, eGFR, and shortened the duration 


\section{Cardio-Renal Anemia (CRA) Syndrome}

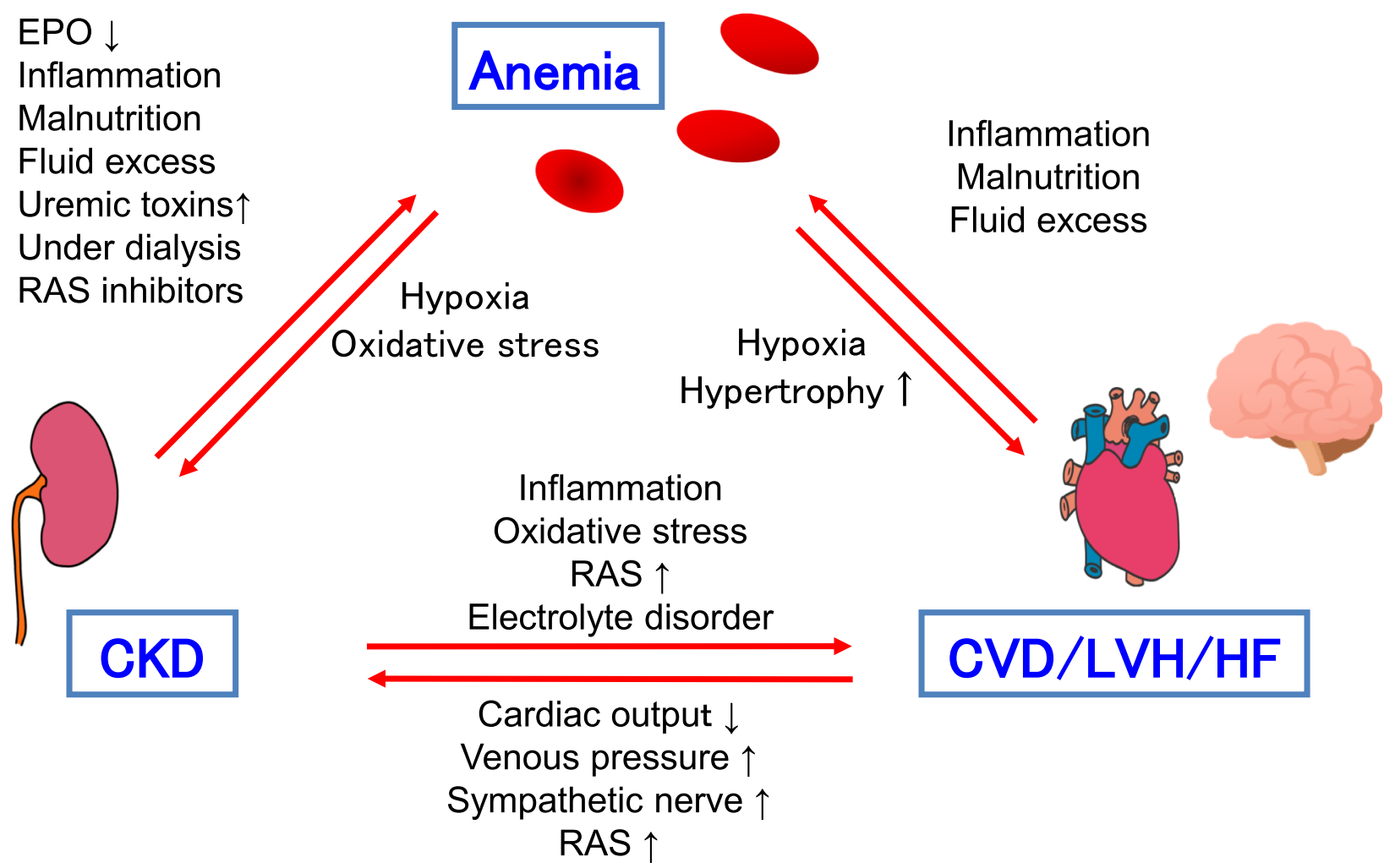

Fig. 1 Cardio-renal-anemia (CRA) syndrome. CKD-induced anemia produces hypoxic condition which leads to an increase in oxidative stress. CKD also facilitates chronic inflammation and hypoxia in renal tissue, activating systemic, and local RAS. These changes trigger to aggravate cardiac hypertrophy and reduce cardiac output, which in turn decreases organ perfusion including the kidney. With such a mechanism, renal anemia in CKD creates a vicious circle in conjunction with CVD/HF, so-called the CRA syndrome, which may eventually result in poor patients' prognosis. CVD cerebrovascular disease, LVH left ventricular hypertrophy, HF heart failure. Quoted from reference \# 23,24

of hospitalization, suggesting that ESA serves as cardiorenal protective [24]. The similar study was conducted by Ayus et al. who confirmed that ESA significantly regressed the LVM index (LVMI) in CKD patients at pre-dialysis stage [25]. After collecting 15 related studies, Parfrey et al. confirmed in a meta-analysis that the regression of LVMI was unequivocally found in the anemia treatment with ESA [1]. Supporting this conclusion is a multicenter study recruiting pre-dialysis patients in Japan that maintaining hemoglobin $(\mathrm{Hb})$ levels above $12 \mathrm{~g} / \mathrm{dL}$ with the use of darbepoetin- $\alpha$ (DA) significantly reduced LVMI [26].

As for the effect of ESA on residual renal function in pre-dialysis patients, early studies showed that the use of ESA retarded the progression of CKD [27, 28]. Recently, Covic et al. performed an extensive meta-analysis on the renal protective effect of ESA and found that there was no benefit when evaluated using the hard-endpoint (EP) such as composite EP including mortality, commencement of dialysis, and renal death. However, when using a doubling of $\mathrm{Cr}$ concentration, the relative risk was as low as 0.53 $(95 \% \mathrm{CI}, 0.31 \sim 0.89)$, suggesting that early intervention with ESA may serve as renal protective [2]. Focusing on renal protection, Akizawa et al. and Tsubakihara et al. also confirmed that the early intervention maintaining the $\mathrm{Hb}$ levels above $12 \mathrm{~g} / \mathrm{dL}$ with ESA was advantageous in protecting the kidney from failing $[29,30]$. On the contrary, a randomized multicenter study by Hayashi et al. in patients with advanced CKD patients with an eGFR of 8 to $20 \mathrm{~mL} / \mathrm{min} /$ $1.73^{2}$ demonstrated that there was no difference in the renal survival between the high $\mathrm{Hb}$-group and the low- $\mathrm{Hb}$ group [31].

Taking all the above mentioned studies into consideration, it can be safely concluded that ESA regresses $\mathrm{LVH}$; thus, serving as cardio-protective, preventing HF and/other cardiac diseases, and eventually contributing to prolonging patients' lives. With respect to renal protection, ESA appears not to have benefits in advanced 
CKD patients, but early intervention with ESA is probably advantageous in retarding the progression of CKD. All in all, the effect of ESA in improving the CRA syndrome appears to be indisputable. Of note is that whether the same holds true with the use of HIF stabilizers remains undetermined, thus needs to be addressed in the future trials.

\section{Factors to explain ESA hyporesponsiveness}

Normal hematocrit cardiac (NHC) trial was the first large-scale RCT to evaluate the effect of ESA in patients on dialysis. Defining death and non-fatal myocardial infarction as the primary EP, comparison was made between the high-hematocrit (Ht) (Ht 42\%) and the low$\mathrm{Ht}$ (Ht 30\%) group. The NHC trial was not completed because of a trend of unexpectedly increased number of death and the excessive amount of iron-supplementation dosage [32]. This study widely provokes a concern that "normalization of Ht with ESA" may not always be necessary for the better outcomes, and thus, setting the target $\mathrm{Ht}$ level in the neighbor of "normal" was in general not recommended. Discussion on the normalization of anemia with ESA was later years extended to patients at pre-dialysis stage in the CREATE, CHOIR, and TREA $\mathrm{T}$ studies. The CREATE showed that therapy with ESA aiming at normal $\mathrm{Hb}$ level had no beneficial effect on the prevention of CV diseases [33]. Second, the CHOIR showed that there was an increasing rate of death, congestive HF, non-fatal myocardial infarction, and cerebral apoplexy [34]. Third, the TREAT study indicated that the incidence of cerebral apoplexy had risen significantly in the high $\mathrm{Hb}$ group [35]. Following these trials, the RED-HF study aiming at the target Hb level above $13 \mathrm{~g} /$ $\mathrm{dL}$ in patients with chronic HF resulted in an increase in the incidence of cerebral thrombosis [36]. The clinical message of these clinical studies is that a targeted $\mathrm{Hb}$ well over $13 \mathrm{~g} / \mathrm{dL}$ or near normal may have an inherent $\mathrm{CV}$ risk that would lead to the poor prognosis of the CKD patients. Notably, post hoc analyses on the CREA TE, CHOIR, and TREAT studies suggested that the true cause for the unexpected adverse events is not the ESA treatment aiming at the high $\mathrm{Hb}$ level per se, but "the ESA hyporesponsiveness" in which there is either high dose of ESA (as a result of weakened response to ESA) or large dose iron supplementation [37-39]. The underlying abnormality to explain ESA-hyporesponsiveness is chronic inflammation and the subsequently accompanying iron dysregulation in CKD patients.

Based on the abovementioned studies, the KDIGO guideline is rather cautious about using ESA. The guideline recommends that iron therapy is the first priority and that ESA should not be the choice even if patient' $\mathrm{Hb}>10 \mathrm{~g} / \mathrm{dL}$. Moreover, it recommends that the target $\mathrm{Hb}$ should be less than $11.5 \mathrm{~g} / \mathrm{dL}$ [40]. However, unlike the KDIGO guideline, the JSDT guideline paid a special attention to set the stage-specific individual target $\mathrm{Hb}$ value, especially distinguishing patients on dialysis from those at pre-dialysis stages. Based on them, the ESA should be started when the $\mathrm{Hb}$ value measured repeatedly is less than $11 \mathrm{~g} / \mathrm{dL}$ if patients are at pre-dialysis stage or on peritoneal dialysis (PD), and it is less than $10 \mathrm{~g} / \mathrm{dL}$ if patients are on hemodialysis (HD). In addition, the targeted $\mathrm{Hb}$ value should be set to $10 \sim 12$ $\mathrm{g} / \mathrm{dL}$ if the patients were on HD and $11 \sim 13 \mathrm{~g} / \mathrm{dL}$ if they were in pre-dialysis stage or on PD [41].

Now that the guideline for HIF stabilizer is not available at this limited point, these values obtained from the studies of ESA should be applicable for the treatment of renal anemia with HIF stabilizers.

As mentioned above, high-dose ESA treatment for anemia in CKD patients might be associated with increased mortality in those patients. With respect to EPO concentration, levels of EPO after administration of ESA is obviously increased compared with those with HIF stabilizers. HIF stabilizers could control renal anemia while keeping EPO within an almost normal physiological range. That could be the advantage in CKD patients with anemia who would be required high dose of ESA for the management.

\section{Dysregulated iron metabolism in CKD patients CKD and MIA syndrome}

The incidence of renal anemia increases linearly as the CKD progresses into its further advanced stage. Recruiting more than 1,000,000 individuals in the in USA in research, Go et al. clearly showed that the progression of renal function to end stage renal disease (ESRD) accelerates risks of death and CV events in an exponential fashion $[42,43]$. What are the hidden mechanisms to create this vicious cycle in CKD? One of the answers to this question is the CRA syndrome, as was discussed previously in this review. But there is another crucial mechanism to cause deterioration of CKD; that is, the abnormal regulation of iron in an association with chronic inflammation. Steinvinkel et al. proposed for the first time the conceptual disease entity of the MIA syndrome [3, 4]. MIA syndrome can be found in most of advanced CKD. Via increase in circulating cytokines such as interleukin (IL)-6, chronic inflammation, and abnormal iron metabolism plays a crucial role in the ESA unresponsiveness and other CKD-accompanying complications in the MIA syndrome. It is conceivable that its pathogenetic mechanisms are more or less overlapped with the CRA syndrome.

\section{Iron sequestration inside of the cells in CKD}

Under the influence of chronic inflammation, secondary to increased inflammatory cytokines and oxidative stress, the iron metabolism is dysregulated in patients with 
CKD. In addition, reactive oxygen species (ROS) formation and production of free radicals due to chronic inflammation also adversely affects iron metabolism [44]. Utilizing polymorphonuclear leukocytes, Otaki et al. for the first time proved clinically that intracellular iron content was increased in patients on dialysis [45], suggesting that iron is sequestrated in the cells. In other words, iron is trapped inside the cells. This mechanism can be accounted for by an increase in hepcidin. Under the circumstances akin to MIA syndrome in CKD, hepcidin synthesis is stimulated in the liver by the increased inflammatory cytokines such as IL-6, and in addition, the reduced renal clearance in CKD also synergistically increases the circulating hepcidin level. Stimulated by iron overload, IL-6, leptin, and the hepcidin downregulate ferroportin-1 (FPN-1) to inhibit iron release from the cells, and at the same time tumor necrosis factor (TNF)-NFolipopolysaccharide (LPS), etc., upregulates transferrin receptor (TfR) and divalent transporter-1 (DMT-1) to trap more iron into the cells [46] (Fig. 2a). Recently, erythroferrone (ERFE) has been identified as a suppressor of hepcidin. Honda et al. demonstrated that ERFE levels are significantly increased with a concomitant decrease in hepcidun-25 by ESA treatment [47]. Hepcidin is involved in iron sequestration inside the cells, and ERFE may be a key regulator which facilitates iron release of the inside the cells into the circulating blood. Since ERFE are produced and released from erythroblasts, ESA or HIF stabilizer acts on the bone marrow to stimulate ERFE and as a result inhibits hepcidin, helping the dysregulated iron mobilization to yield better outcomes [48]. Interestingly, HIF could regulate FPN-1 and hepcidin production directly in non-ERFEdependent pathway. That is, the von Hippel-Lindau/ hypoxia-inducible transcription factor (VHL/HIF) pathway is an essential link between iron homeostasis and hepcidin regulation in vivo. Through coordinate downregulation of hepcidin and upregulation of erythropoietin and FPN-1, the VHL-HIF pathway mobilizes iron to support erythrocyte production [49].

All in all, iron metabolism in renal anemia of CKD is characterized by increase in hepcidin due to the increased inflammatory cytokines. Iron in CKD is not deficient, and there is rather abundant iron inside the cells. Nevertheless, functionally deficient iron outside of the cells is not sufficient enough to elicit efficient erythropoiesis. So, this is a paradoxical regulation of iron where "Excessive iron is present inside the cells but, functionally iron is deficient outside the cells". Incidentally, elevation of EPO by either ESA or HIF stabilizer lowers

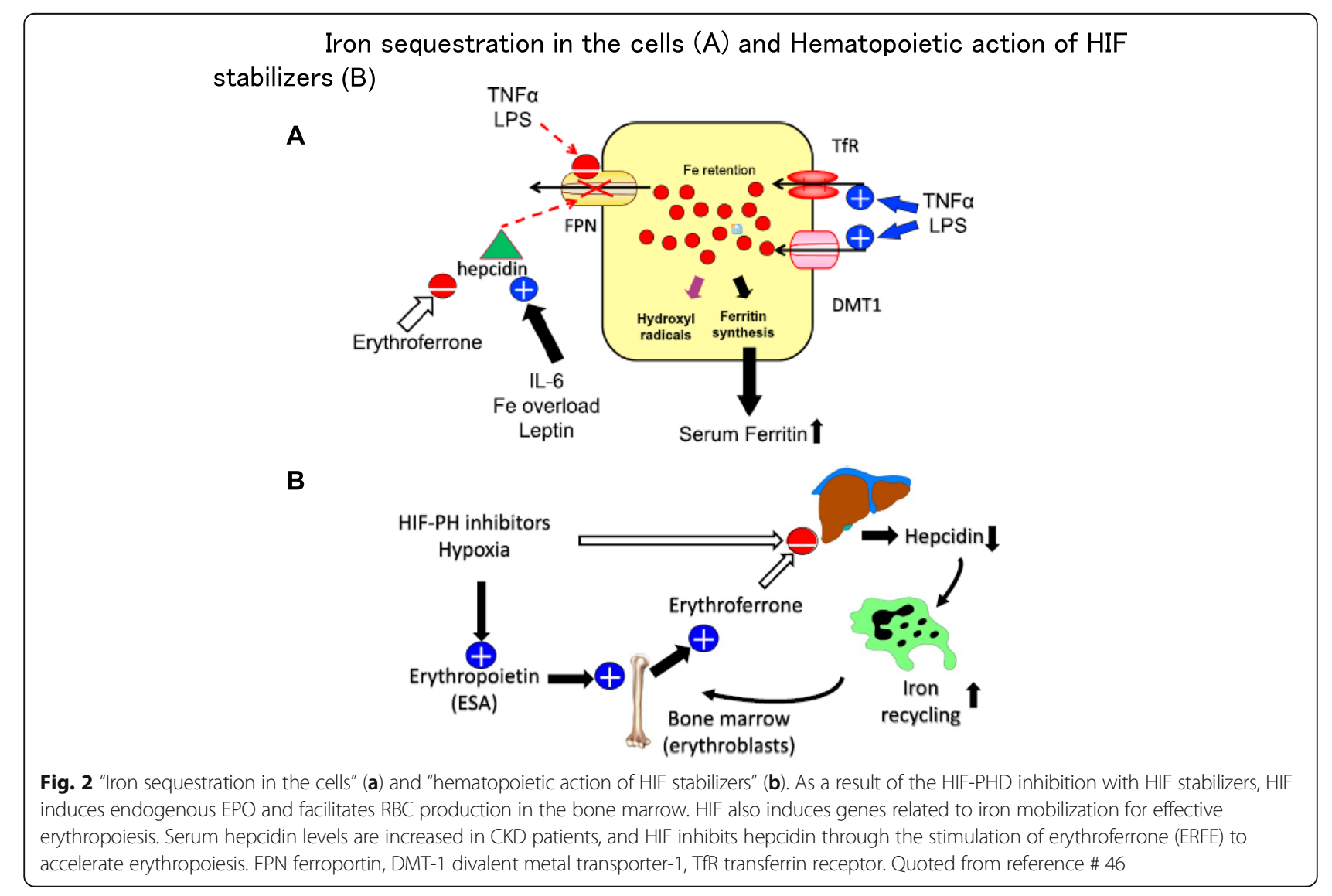


hepcidin level and improves the utilization of iron more effectively through the increase in ERFE [46] (Fig. 2b).

\section{Serum ferritin and patients' mortality}

Serum ferritin concentration is crucial in the determination of a patient's prognosis. It is known that the higher the serum ferritin concentration is, the poorer the patients' mortality becomes [50]. Serum ferritin levels are substantially different country by country. It is 83 $\mathrm{ng} / \mathrm{mL}$ in Japan, $405 \mathrm{ng} / \mathrm{mL}$ in Europe, and $718 \mathrm{ng} / \mathrm{mL}$ in the USA [51]. The similar trend has been found in two other Japanese studies in which serum ferritin levels were relatively low of $73 \mathrm{ng} / \mathrm{mL}$ in $\mathrm{HD}$ patients [52], and the rate of patients with ferritin levels > $500 \mathrm{ng} / \mathrm{mL}$ accounted for only $11.4 \%$ in a separate study population [53]. Of note is that Hamano et al. investigated thresholds of iron markers for iron deficiency erythropoiesis and suggested that the patient subgroup with TSAT < $20 \%$ and ferritin $>100 \mathrm{ng} / \mathrm{mL}$ had significantly higher ERIs compared with the subgroup with TSAT $>20 \%$ and ferritin < $100 \mathrm{ng} / \mathrm{mL}$, implying that TSAT, rather than ferritin, should be a primary iron marker predicting ESA response [53].

Coincidentally, the mortality is the lowest in Japan, followed by Europe and the USA in this order, suggesting a possibility that higher ferritin levels can be associated with worse outcomes. Japan is a leading country when it comes to the outcomes of dialysis treatment. Death risk in dialysis patients is 1 (a reference) in Japan, 2.4 in Europe, and 2.8 in the USA, which is in good accordance with the abovementioned patients' ferritin status [54]. Using the baseline data of 191,902 patients on dialysis, Maruyama et al. performed the nationwide survey on the relationship between serum ferritin and mortality. The study found that higher baseline serum ferritin levels were associated with higher mortality rate among patients undergoing HD [55]. Nevertheless, the reason why Japan leads Europe and the USA is not simply because the iron management therapy in Japan is superior to other countries. It may be influenced by multiple factors and reasons, i.e., health insurance systems, reimbursement system, differences in dialysis modality, quality of dialysis technique, the availability of A-V fistula, quantity of blood flow, and patients' adherence to the therapy.

\section{Appropriate iron supplement therapy}

Excessive iron overload may have a deleterious effect on life prognosis even in individuals without CKD. Notably, in Iowa women's health study recruiting 38,772 women in the USA, the hazard risk was higher in individuals who consumed iron supplement for a longer period and a larger amount [56]. Because iron metabolism is a closed reuse circuit without efficient excretion pathway, iatrogenic excessive iron overload will easily result in excessive iron burden in humans. Rostoker et al. using MRI demonstrated that HD patients whose serum ferritin level exceeded $290 \mathrm{ng} / \mathrm{mL}$ frequently have serious iron deposition in the liver [57]. Through the observational study in patients on HD, Ogawa et al. recommended that an appropriate criterion in iron management might be "serum ferritin $<90 \mathrm{ng} / \mathrm{mL}$ and TSAT < 20\%" [58]. In addition, Anraku et al. found that acute and frequent administration of intravenous iron induced a substantial increase in oxidized albumin and serum ferritin level, suggesting that the iron supplement plan should be carefully managed [59]. Based on these studies, the JSDT takes these studies into consideration and creates a guideline, recommending the moderate use of iron. The JSDT guideline suggests that iron supplementation has priority when serum ferritin level $<50 \mathrm{ng} / \mathrm{mL}$ in ESA naïve patients. The guideline also suggests iron therapy for patients who are treated with ESA and cannot maintain target $\mathrm{Hb}$ levels if both the following conditions are satisfied: absence of disease that decreases iron utilization rate; and serum ferritin level $<100 \mathrm{ng} / \mathrm{mL}$ or TSAT $<20 \%$. On the other hand, iron therapy is not recommended when ferritin level $\geq n 300 \mathrm{ng} / \mathrm{mL}$ [60].

Compared to the JSDT guideline which imposes relatively strict restriction on the use of iron, overseas guidelines are affirmative to allow a relatively high-dose iron supplementation. In the PIVOTAL study in which patients on maintenance dialysis were randomly assigned to the two dose of iron, Macdougall et al. demonstrated that the high-dose intravenous iron administration $(400$ $\mathrm{mg}$ /month) improved death rate, $\mathrm{CV}$ event, and reduction in blood transfusion treatment [61]. Moreover, there was no difference in the prevalence of infection between the high dose $(400 \mathrm{mg} / \mathrm{month})$ and the low dose (100 mg/month) [62]. These results may support an argument that iron supplementation might be required even though patients' ferritin levels exceed $200 \sim 300$ $\mathrm{ng} / \mathrm{mL}$. Similarly, Coyne and Fishbane insisted that an iron status of "serum ferritin around $200 \mathrm{ng} / \mathrm{mL}$ and TSAT $<20 \%$ " is regarded as a functional iron deficiency and that iron supplementation should be recommended [63]. This concept of relatively high dose of iron therapy has been supported by the other oversea researchers that even if serum ferritin level exceeds $700 \mathrm{ng} / \mathrm{mL}$ under the condition of ESA resistance, the iron supplementation is still acceptable. An AIM-HD trial, performed in Taiwan recruiting 42,230 HD patients during 2001 to 2008, demonstrated that patients with $\mathrm{Hb}$ levels lower than $10 \mathrm{~g} / \mathrm{dL}$ had a higher mortality [64]. In contrast, the AIM-HD found that those having ferritin level of $300 \sim 800 \mathrm{ng} / \mathrm{mL}$ and TSAT of $30 \sim 50 \%$ had a lower mortality, and this also defends that the high-dose iron may be acceptable. Incidentally, according to the KDIGO guideline, iron 
therapy is the first priority even if patients' iron status is TSAT $30 \%$ and serum ferritin $500 \mathrm{ng} / \mathrm{mL}$ [40].

Although at this point, there is no direct clinical evidence that excessive iron load would lead to poor patients' prognosis, one must be alert to the possibility that careless and aimless iron supplementation is far physiological considering that the regulatory system of iron is a closed circuit. One must also be aware of a fact that even without ESA or HIF stabilizers, iron replacement therapy, per se, at least in part, improves renal anemia. But that does not necessarily imply that the treatment is scientifically justified. Physicians must recognize that there is "A pitfall in iron therapy in CKD"; CKD is unequivocally associated with "Sequestration of iron in the cells with functional iron deficiency outside the cells". After all, iron therapy should also be considered on the premise that the use of either ESA or HIF stabilizers improves the dysregulated iron metabolism via the hepcidin-ERFE axis.

\section{Clinical significance of HIF stabilizers Development of HIF stabilizers}

Now that we come to understand the pathophysiology of renal anemia and its therapeutic interventions through the experiences with ESA, the latter part of this article will deal with how HIF stabilizer will be applied for CKD in our daily clinical practice.

Given that high altitude training improves physical performance of athletes, continuous exposure to hypoxia stimulates endogenous EPO. The similar effect is expected even in patients with impaired renal function. Focusing on the relationship between land altitude and the death rate of inhabitants who were on dialysis, Winkelmayer et al. performed a large-scale observational study recruiting 80 , $000 \mathrm{HD}$ patients for a period of 20 years. The cohort suggests that those who live in high lands over $1800 \mathrm{~m}$ in altitude had a lower relative death rate by $15 \%$ [65]. It implies that even if kidney function is severely damaged, it responds well to the altitude-dependent hypoxic condition.

In CKD, glomerular sclerosis, interstitial fibrosis, and vascular lesions accelerate hypoxia in the kidney. Under these abnormal conditions, the activation of HIF is not sufficient to induce the secretion of endogenous EPO from the EPO-producing (REP) cells, resulting in renal anemia. HIF stabilizer is an inhibitor of HIF-PHD which stimulates endogenous EPO by mimicking hypoxia with HIF-PHD inhibition [66]. For the first time in 30 years since ESA became available in 1990, HIF stabilizer has been newly launched into the treatment of renal anemia. Of note is that circulating endogenous blood concentration of EPO produced by HIF inhibition are within a physiological level (the peak is about $100 \mathrm{mU} / \mathrm{mL}$ at most). In contrast, exogenously given ESA produces a so-called overshooting of EPO concentration (the peak is about $500 \mathrm{mU} / \mathrm{mL}$ or more) (Table 1). It is of particular interest by inferring from this fact that EPO-induced hypertension with the use of ESA may be relevant to increase in unphysiologically high EPO concentration which acts on VSMC to constrict the vasculature, leading to increase in blood pressure (BP).

\section{Characteristics of HIF stabilizers}

In November 2019, the novel drug for renal anemia, Roxadustat, became available for the first time in Japan. At the beginning, its indication was only limited to renal anemia in patients on dialysis. Subsequently, as of August 2020, daprodustat and vadadustat become available, which are indicated for both patients on dialysis and those in pre-dialysis stage. The indication of roxadustat will be extended to patients at pre-dialysis stage by the end of this year. The other two new agents, molidustat and enarodustat, will also be available soon.

The pharmacological potency of these HIF stabilizers to ameliorate renal anemia may be different because each has its own different pharmacological property in the pharmacokinetics $(\mathrm{PK})$ and the pharmacodynamics (PD) (Cmax, T1/2, Tmax, AUC, Ki, IC50). Molecular and cellular mechanisms of HIF stabilizers have been reviewed by Yeh et al. that there are substantial differences in the in vitro characterizations of pharmacological properties, PK, and PD of HIF inhibition among different HIF stabilizers [67].

The notable difference between ESA and HIF stabilizer is the route of administration (Table 1). ESA is given either subcutaneously or intravenously, whereas HIF stabilizer is given orally. Because of the clinical problem of polypharmacy in which CKD patients are normally given multiple drugs, whether the oral use is more advantageous than the other still needs to be further evaluated. Other potential merits of choosing HIF

Table 1 Clinical characteristics of ESA and HIF stabilizer

\begin{tabular}{llllll}
\hline Drug & EPO origin & EPO concentration at peak & Route & Correction of anemia & Adverse events \\
\hline ESA & Exogenous & High (overshooting) & IV or SC & Correction of anemia & Hypertension thrombosis \\
HIF-S & Endogenous & Within physiological levels & Oral & Effective (especially, nondialysis) & $\begin{array}{l}\text { Thrombosis/embolism pulmonary } \\
\text { hypertension/ADPKD/malignancy/ } \\
\text { retinopathy/hyperkalemia } \\
\text { hypertension? }\end{array}$ \\
& & & & & \\
\hline
\end{tabular}


stabilizers include probably low cost, improved iron profile, and endogenous EPO at levels close to physiological range.

Of note is that there are a lot of target genes of HIF activation (approximately $100 \sim 200$ genes); thus, the gene-dependent unwanted pharmacological effects should be taken into consideration. At this point, however, there is no precise information on the class effects, the drug effects, and the adverse effects of HIF stabilizers. The followings are the main results of HIF stabilizers obtained in the phase- 2 and phase- 3 clinical studies that may provide us some hints to differentiate one from the others.

Daprodustat Based on the phase- 2 trials in patients on $\mathrm{HD}$ and those at pre-dialysis stage, daprodustat increased $\mathrm{Hb}$ levels in a dose-dependent manner compared to the placebo and the rHuEPO-treated group [8-11]. In a phase3 trial, Tsubakihara et al. demonstrated that daprodustat significantly increased $\mathrm{Hb}$ value up to $10 \sim 12 \mathrm{~g} / \mathrm{dL}$ in the ESA-naïve patients. This effect was accompanied by the reduction in hepcidin, ferritin, and TSAT with a subsequent increase in TIBC [68]. In addition, Akizawa et al. also found that daprodustat increased $\mathrm{Hb}$ in a dose-dependent manner in $\mathrm{HD}$ patients having anemia ranging $8.5 \sim 10.5 \mathrm{~g} / \mathrm{dL}$ (average $9.8 \mathrm{~g} / \mathrm{dL}$ ). Furthermore, this study found that VEGF remained unchanged, and the circulating endogenous EPO remained within a physiological range with a few exceptional cases whose EPO levels were as high as the ones observed in the ESA-treated [10]. Other reports showed that daprodustat increased $\mathrm{Hb}$ in a dose-dependent manner in $\mathrm{HD}$ patients whose pretreatment average $\mathrm{Hb}$ value was less than $10 \mathrm{~g} / \mathrm{dL}$, and the secondary analysis showed that VEGF remained unchanged and the endogenous EPO concentration was within a physiological range (maximum EPO concentration $<50 \mathrm{mIU} / \mathrm{mL}$ ). As for ESA resistance, Akizawa et al. found that darbepoetin- $\alpha \mathrm{rDA}$ ), compared to daprodustat, required a broader range administration dose, suggesting that the ESA hyporesponsiveness is better controlled in the daprodustat-treated group [69]. In addition, there was basically no difference in the PK and the PD between Caucasian and Japanese with a slightly high AUC in the latter, which may be originated from the difference in body size [70]. The protein binding is about $99 \%$, and thus, the drug is not dialyzable. The T1/2 is estimated to be $1.3 \sim 2.5 \mathrm{~h}$.

Roxadustat The phase- 2 and phase- 3 randomized clinical studies clearly demonstrated that roxadustat was effective in ameliorating renal anemia in all patient groups; pre-dialysis, HD, and PD [71-73]. Interestingly, Provenzano et al. compared roxadustat to epoetin- $\alpha$ to investigate how inflammation affects drug responses. They found that the epoetin- $\alpha$-treated group required larger dosage to maintain $\mathrm{Hb}$ levels in patients having higher CRP, whereas such a dose dependency was not found in the roxadustat-treated group [6]. Chen et al. reported that patients treated with epoetin- $\alpha$ had lower and slower response to attain the target $\mathrm{Hb}$ especially in patients with higher CRP. In contrast, roxadustat required smaller dosage to attain the target $\mathrm{Hb}$ value, irrespective of CRP levels [74]. These results of roxadustat are suggestive that HIF stabilizer may be more beneficial than ESA in maintaining the target Hb levels even in the presence of chronic inflammation. In fact, roxadustat unequivocally reduced hepcidin and ferritin levels $[6$, 72-74]. More interestingly, increase in EPO concentration in response to the treatment was significantly lower in the roxadustat-treated (maximum EPO concentration $<130 \mathrm{mIU} / \mathrm{mL}$ ) than in the epoetin- $\alpha$-treated (maximum EPO concentration $<700 \mathrm{mIU} / \mathrm{mL}$ ), suggesting a benefit of choosing the former to prevent, for instance, an abrupt increase in BP due to ESA, so-called EPOinduced hypertension [6].

Lowering effects of total- and LDL-cholesterol concentration has been reported in the phase-3 trial [74]. In terms of adverse events, there were less incidence of hypertension and hyperkalemia compared to the rHuEPO-treated $[73,74]$. The T1/2 is estimated to be 12 $\sim 15 \mathrm{~h}$. The protein binding is sufficiently high; thus, the drug is not dialyzable.

Vadadustat A phase-2 study in patients at pre-dialysis stage demonstrated that vadadustat improved renal anemia compared to the placebo [18]. There was a significant reduction in hepcidin and ferritin accompanied by an increase in TIBC, while CRP, VEGF, total cholesterol, and BP remain unchanged. Another phase-2 study demonstrated that $\mathrm{Hb}$ levels were well maintained with vadadustat after the pre-treatment with epoetin- $\alpha \mathrm{p} 12$. Serum iron was slightly increased; hepcidin, ferritin, and TSAT remained unchanged; and TIBC was increased. No serious adverse events were observed [12]. Noteworthy is that the phase- 2 study recruiting 210 patients at pre-dialysis stage, cystatin-C, was significantly lowered in the vadadustat-treated group than in the placebo group (vadadustat group $34.9 \mathrm{ng} / \mathrm{mL}(n=138)$ vs. placebo: $298.2 \mathrm{ng} / \mathrm{mL}(n=72))$, suggesting that vadadustat may provide a renal protective effect [17]. Whether this agent contributes to retard the progressive lowering of residual renal function in non-dialysis patients needs to be evaluated. Interestingly, the dose-response curve of HIF inhibition showed that the \%inhibition with vadadustat had the most gentle slope among other HIF stabilizers, suggesting a slow and mild pharmacological action that may be essential to avoid abrupt increase in $\mathrm{Hb}$ value in the treatment of renal anemia [67]. The T1/ 2 of vadadustat is estimated to be $7 \sim 9 \mathrm{~h}$, and the effect is not influenced by HD treatment. 
Currently, the internationally organized large-scale studies, the INNO2VATE, and PRO2TECT trials are ongoing. The former makes a prompt news, reporting the non-inferiority of vadadustat to DA with respect to the effect on anemia correction and the incidence of major cardiovascular events (MACE). The results of the INNO2VATE will be announced sometime soon.

Molidustat In a phase-2 study, Macdougall et al. reported that there was a dose-dependent increase in $\mathrm{Hb}$ levels in the moridustat-treated group and the effect was better than that in the placebo, the DA-treated, and the rHuEPO-treated groups [13]. Notably, patients on dialysis required higher dosage. In patients at pre-dialysis stage, molidustat reduced hepcidin, ferritin, and increased TIBC. However, in patients on dialysis who were previously treated with ESA, the ferritin, TSAT, and iron were increased instead. There was a slight decrease in LDL cholesterol concentration. As an extension of the former phase-2 study, Akizawa et al. confirmed that molidustat is effective in improving renal anemia in both patients on HD and those at pre-dialysis stage [13, 75]. Furthermore, hepcidin in the molidustat-treated was lowered more efficiently than that in the DA-treated in patients at pre-dialysis stage [76]. Although there were some cases with thrombosis/embolism, their symptoms were all mild. As far as the effect of molidustat on BP level, only small number of cases showed mild elevation of BP, all of whom were not clinically problematic. In addition, only a few cases with hyperkalemia were reported [13]. Currently, a phase-3 study, named the MIYABI study, is ongoing in Japan which aims at evaluating the effect of anemia correction of molidustat in patients on HD, PD, and those at pre-dialysis stage. The result will be announced sometime within this year, 2020.

Enarodustat Akizawa et al. investigated the effect of enarodustat in CKD patients at pre-dialysis stage in a double-blind placebo phase-2 trial [19]. The study showed that enarodustat increased $\mathrm{Hb}$ in a dosedependent fashion. Analyses of iron regulation showed that ferritin, hepcidin, and TSAT were lowered, whereas TIBC was increased. The incidence of hypertension was $8.5 \%$ in the enarodustat naïve group and $4.9 \%$ in the conversion group (from ESA) group. The prevalence of hyperkalemia was $3.2 \%$ in the naïve group and $5.8 \%$ in the conversion group. No other serious adverse events were observed [77]. In another phase- 2 study in HD patients, VEGF remained unchanged throughout the study period [19]. The T1/2 of enarodustat is $15 \mathrm{~h}$.

The results of phase- 3 study were announced in the American Society of Nephrology (ASN) in 2019, and the related matters will soon be published. The basic message in the ASN was the non-inferiority of enarodustat to DA in both patients on dialysis and those at pre-dialysis stage.

\section{Meta-analysis of HIF stabilizers}

Collecting 12 clinical studies, Zhong et al. summarized the effect of HIF stabilizers in the meta-analysis [78]. In general, the improvement of renal anemia is more apparent in the pre-dialysis group than in the dialysis group. It implies that patients having residual renal function may have a greater capacity to produce endogenous EPO from the kidney in response to HF inhibition treatment. Hasse et al. focused on the iron metabolism in CKD patients and summarized that HIF stabilizers reduced ferritin, hepcidin, and TSAT with the concomitant increase in TIBC, proposing that these changes are "the class effect of HIF stabilizers" [79].

Although the precise comparative study is not available at this point, whether lowering of hepcidin in patients at pre-dialysis stage is superior to that in dialysis patients or not is of particular interest. Based on the speculation from the abovementioned previous meta-analyses, it appears that hepcidin lowering effect is more efficacious in predialysis patients than in those on dialysis $[78,79]$.

\section{Adverse events of HIF stabilizers}

Ultimately, clinical evaluation of adverse reactions should be carried out with a focus on the major cardiovascular events (MACE). In a pooled phase 3 data of the placebo-based, double-blind studies consisting of 4270 non-dialysis patients, the risks of MACE (death, apoplexy, and myocardial infarction), MACE+ (MACE + unstable angina and/or CHF requiring hospitalization), and overall death were comparable between the roxadustattreated and the placebo. In a pooled data in 3880 patients on dialysis, the risks of MACE and overall death were comparable between the roxadustat-treated and theotepoetin-treated. However, the risk of MACE+ in the roxadustat-treated was significantly lower than that in the $\alpha$-epoetin-treated (HR 0.86, 95\%CI $0.74 \sim 0.98$ ). Furthermore, in 1526 patients who were newly treated with dialysis, the risk of MACE (HR 0.70, 95\%CI 0.51 $0.96)$ and MACE+ (HR 0.66, 95\%CI $0.50 \sim 0.89)$ were lower in the roxadustat-treated than in the $\alpha$-epoetintreated [80].

The target genes on HIF activation are estimated as much as $100 \sim 200$, theoretical fear that some of them may bring unfavorable reactions is inevitable. Such assumptive events of HIF stabilizers include thromboembolic (TA) event, relapse of cancer, hypertension, pulmonary hypertension, worsening of retinopathy, hyperglycemia, and BP elevation [81].

Despite theoretical fear, no serious TA events and few cases of the arterio-venous shunt failure have been reported in the treatment with roxadustat [71], daprodustat 
[10], vadadustat [12, 17, 18], molidustat [13], and enarodustat [19]. Another concern is the potential involvement of increased VEGF which may induce worsening of diabetic retinopathy, vascular neogenesis of malignant tumor, and progression of autosomal dominant polycystic kidney disease (ADPKD). Most of the clinical studies suggested that VEGF was unchanged $[9,10,18,77]$, but there may be a slight elevation at a higher dosage. HIF stabilizer may have an adversely effect on pulmonary hypertension $(\mathrm{PH})$, since the prevalence of $\mathrm{PH}$ is higher in CKD and the pathogenesis of PH may have a link to HIF-2. As far as hypertension is concerned, compared to ESA, which is frequently associated with an increase in BP, the net effect of HIF stabilizers on BP appears not so problematic. The prevalence of hypertension was reported to be $10 \%$ in the overall roxadustat-treated groups [5], and $8.5 \%$ in the enarodustat-treated group [77], both of which were not placebo-based comparisons. Hypertension was found 8\% in the vadadustat-treated patients, which was lower than the rates in the ESA-treated patients [17]. Interestingly, molidustat lowered BP through the inhibition of RAS in an experimental model [82]. All of the above findings are supportive that the effect of HIF stabilizers on BP appears to be neutral. In many animal experiments in general, HIF stabilizers have been proven renal protective [66].

Whether there is any association of malignant tumors with the clinical use of HIF stabilizers is a matter for debate. So far, no clinical studies are suggestive such a relationship [11, 16-18], and one of the main reasons is that the observation periods were too short.

An experiment using rats showed that the teratogenicity was found in the fetus; therefore, one must be alert to the possibility that HIF stabilizer may not indicated for pregnant women and those who are willing to be pregnant.

Most of the studies are supportive that HIF stabilizers reduced total and LDL cholesterol levels [10, 13, 15, 73]. The effect on total cholesterol and TG was neutral with vadadustat [18].

As for drug interactions, the effect of HIF stabilizers can be weakened with the co-administration of the phosphate binding polymers such as sevelamer and uric acid lowering agent, probenecid. On the contrary, the effect may be potentiated with the combined use of lipid-lowering agent, statin.

\section{Is HIF stabilizer alternative to ESA?}

\section{Perspectives on the indications of HIF stabilizers}

The issue of whether ESA can be fully or partially replaced by HIF stabilizer in the future treatment is worth debating, but at this point, there is no appropriate guidelines except for the recommendation released by the Japanese Society of Nephrology (JSN) in the 29th of September 2020 [83]. By taking this newly available recommendation into consideration and by having what we obtained in the clinical practice on ESA thoroughly reconsidered, we gain a new insight into how to use HIF stabilizers. The followings are our opinion-based recommendation on the positive indications of HIF stabilizers (Table 2). There may be 3 major practical indications which are summarized as the following:

1) CRA syndrome aiming at more efficient cardio-renal protection (especially, at pre-dialysis stage)

2) ESA-resistant anemia or ESA-unresponsiveness (especially, at dialysis stage)

3) MIA syndrome and/or the neighborhood of iron dysregulated state (especially, at dialysis stage)

These assumptive proposals listed above, especially [2] and [3], are both closely related to the dysregulation iron metabolism in CKD. We feel that it is of importance on choosing HIF stabilizers; evaluation of the iron status is indispensable. Seeking the possible causes for ESAunresponsiveness is also crucial.

We would like to make sure that our listed indications above do not imply that we only insist the indication of "conversion from ESA therapy to HIF stabilizers therapy". Needless to say, when facing the commencement of renal anemia therapy (either ESA or HIF stabilizer naive case), either of them in conjunction with an appropriate iron therapy is a decent option. To fulfil this purpose, early pharmacological introduction of the above may have a significant contribution to prevent both the CRA syndrome and the accompanying MIA syndrome [1-4, 23, 24].

Aside from the above positive indications, consideration should be paid to avoid the HIF stabilizer-related

Table 2 Positive indications and conditions requiring clinical consideration (opinion-based recommendations of HIF stabilizers)

\footnotetext{
Positive indications

1. ESA resistance or unresponsiveness

2. Cardio-renal protection in CRA syndrome

3. Iron mobilization in the condition akin to MIA

syndrome

Consideration required

1. Thrombosis and/or embolism

2. Malignancy and retinopathy

3. Pulmonary hypertension

4. ADPKD

5. Pregnancy or desire to bear children

6. Hyperkalemia, liver dysfunction

Note:The adverse effects of ESA are already well known; however, those of HIF stabilizers have not always been clarified clinically. The adverse effects with HIF stabilizers include expanded interpretations of the ESA-based findings and/or the inferences based on animal experiments

ESA erythropoiesis stimulating agent, MIA malnutrition-inflammationatherosclerosis, CRA cardiovascular-renal-anemia, ADPKD autosomal dominant polycystic kidney disease
} 
putative adverse events. Most of these unknown clinical adverse events have not yet been virtually clarified. Indeed, most of them are only suggested in experimental animal studies. Such adverse events include thrombosis/ embolism, relapse of the latent cancer, worsening of pulmonary hypertension, autosomal dominant polycystic kidney disease (ADPKD), and diabetic nephropathy et al. (Table 2). Clinical information on these fearful adverse effects with HIF stabilizers is scant at this point, since the observation period in the most studies are too short to draw any scientific conclusion. Regarding hypertension, because no serious BP elevation has been found in patients treated with HIF stabilizers, an insight into whether ESA-induced hypertension is improved with the alternative use of HIF stabilizers or not is of particular interest.

\section{Evidence-practice-gap}

The Japan Chronic Kidney Disease Database (J-CKDDB) is a large-scale, nationwide comprehensive clinical database of patients with CKD. Recruiting 31,082 CKD patients staging $3-5$ in 7 university hospitals. The JCKD-DB cohort discloses that the rate of Hb levels with optimal ranges defined as $\mathrm{Hb}$ value $\geq 11 \mathrm{~g} / \mathrm{dL}$ without ESA or $11<\mathrm{Hb}$ value $<13 \mathrm{~g} / \mathrm{dL}$ with ESA in the CKD stages 4 and 5 is estimated $51.7 \%$. Notwithstanding this high incidence, the utilization of ESA is limited to only $12.1 \%$, suggesting that the recognition and treatment of renal anemia among physicians in clinical practice is still low and inadequate [84]. This evidence-practice-gap can be solved by introducing early use of either ESA or HIF stabilizer with an appropriate iron management.

Finally, our personal opinions about the best way to treat renal anemia are as the following: (1) start the treatment with oral iron supplementation, (2) coadminister one of the HIF stabilizers, (3) evaluate the iron status periodically, and (4) adjust their dosages carefully. By doing so, one can accomplish slow and stable supply of both iron and the effective erythropoiesis that will be maximally beneficial not only for the correction of anemia but for the prevention of $\mathrm{CV}$ events and the dysregulation of iron metabolism.

\section{Conclusion}

The novel anti-anemic agent HIF stabilizer has been evaluated through the clinical lessons of ESA obtained in the past 3 decades. HIF stabilizer will be primarily indicated for CRA syndrome, ESA-resistant anemia, and MIA syndrome. Multiple other factors such as medicoeconomical consideration, patients' preference and adherence, management of concurrent complications, drug interactions, and unwanted adverse events should further be taken into consideration.

\section{Abbreviations}

AUC: Area under the blood concentration curve; A-V fistula: Arterio-venous fistula; CKD: Chronic kidney disease; $C_{\max }$ : Maximum drug concentration; eGFR: Estimated glomerular filtration rate; ERI: ESA resistance index; Hb: Hemoglobin; Ht: Hematocrit; IL-6: Interleukin-6; IC50: Half maximal (50\%) inhibitory concentration; JSDT: Japanese Society of Dialysis Treatment; JSN: Japanese Society of Nephrology; KDIGO: Kidney Disease Improving Global Outcomes; Ki: Affinity coefficient of the drug; MDS: Myelodysplastic syndrome; NYHA: New York Heart Association functional classification; QOL: Quality of life; RCT: Randomized control trial; T1/2: Elimination half-life; $T_{\max }$ : Time to maximum concentration; TIBC: Total iron binding capacity; TSAT: Transferrin saturation; VSMC: Vascular smooth muscle cells

\section{Authors' contributions \\ SK contributed to the overall concept and study design. SK, YM and HH contributed to the intellectual discussion during manuscript drafting, revision, and the approval of the final version.}

\section{Funding}

None

Ethics approval and consent to participate Not applicable

Consent for publication

Not applicable

\section{Competing interests}

The authors declare that they have no competing interests.

\section{Author details}

${ }^{1}$ Jikei University School of Medicine, 3-25-8, Nishi-shinbashi, Minato-ku, Tokyo 105-8471, Japan. ${ }^{2}$ Nephrology \& Hypertension Research Unit, Internal Medicine, Miho Clinic, Shin-Osaki-kangyo Bld 2F, Osaki, Shinagawa-ku, Tokyo 141-0032, Japan. ${ }^{3}$ Division of Nephrology \& Hypertension, Department of Internal Medicine, Jikei University School of Medicine, 3-25-8, Nishishinbashi, Minato-ku, Tokyo 105-8471, Japan. ${ }^{4}$ Department of Internal Medicine, Division of Nephrology, Showa University School of Medicine, 1-5-8, Hatanodai, Shinagawa-ku, Tokyo 142-8555, Japan.

Received: 7 October 2020 Accepted: 30 November 2020

Published online: 09 December 2020

\section{References}

1. Parfrey PS, Lauve M, Latremouille-Viau D, Lefebvre P. Erythropoietin therapy and left ventricular mass index in CKD and ESRD patients: a meta-analysis. Clin J Am Soc Nephrol. 2009;4(4):755-62. https://doi.org/10.2215/CJN. 02730608 Epub 2009 Apr 1.

2. Covic A, Nistor I, Donciu MD, Dumea R, Bolignano D, Goldsmith D. Erythropoiesis-stimulating agents (ESA) for preventing the progression of chronic kidney disease: a meta-analysis of 19 studies. Am J Nephrol. 2014; 40(3):263-79. https://doi.org/10.1159/000366025 Epub 2014 Oct 15.

3. Stenvinkel P, Heimburger O, Paultre F, Diczfalusy U, Wang T, Berglund L, et al. Strong association between malnutrition, inflammation, and atherosclerosis in chronic renal failure. Kidney Int. 1999:55:1899-911.

4. Stenvinkel P. Inflammatory and atherosclerotic interactions in the depleted uremic patient. Blood Purif. 2001;19:53-61.

5. Besarab A, Chernyavskaya E, Motylev I, Shutov E, Kumbar LM, Gurevich K, et al. Roxadustat (FG-4592): correction of anemia in incident dialysis patients. J Am Soc Nephrol. 2016;27:1225-33.

6. Provenzano R, Besarab A, Wright S, Dua S, Zeig S, Nguyen P, et al. Roxadustat (FG-4592) versus epoetin alfa for anemia in patients receiving maintenance hemodialysis: a phase 2, randomized, 6- to 19-week, openlabel, active-comparator, dose ranging, safety and exploratory efficacy study. Am J Kidney Dis. 2016;67:912-24.

7. Chen N, Qian J, Chen J, Yu X, Mei C, Hao C, et al. Phase 2 studies of oral hypoxia-inducible factor prolyl hydroxylase inhibitor FG-4592 for treatment of anemia in China. Nephrol Dial Transplant. 2017:32:1373-86.

8. Holdstock L, Meadowcroft AM, Maier R, Johnson BM, Jones D, Rastogi A, et al. Four-week studies of oral hypoxia-inducible factor-prolyl hydroxylase 
inhibitor GSK1278863 for treatment of anemia. J Am Soc Nephrol. 2016;27: 1234-44.

9. Brigandi RA, Johnson B, Oei C, Westerman M, Olbina G, de Zoysa J, et al. A novel hypoxia-inducible factor-prolyl hydroxylase inhibitor (GSK1278863) for anemia in CKD: a 28-day, phase 2a randomized trial. Am J Kidney Dis. 2016; 67:861-71.

10. Akizawa T, Tsubakihara Y, Nangaku M, Endo Y, Nakajima H, Kohno T, et al. Effects of daprodustat, a novel hypoxia-inducible factor prolyl hydroxylase inhibitor on anemia management in Japanese hemodialysis subjects. Am J Nephrol. 2017:45:127-35.

11. Meadowcroft AM, Cizman B, Holdstock L, Biswas N, Johnson BM, Jones D, et al. Daprodustat for anemia: a 24-week, open-label, randomized controlled trial in participants on hemodialysis. Clin Kidney J. 2019;12:139-48.

12. Haase VH, Chertow GM, Block GA, Pergola PE, DeGoma EM, Khawaja Z, et al. Effects of vadadustat on hemoglobin concentrations in patients receiving hemodialysis previously treated with erythropoiesis-stimulating agents. Nephrol Dial Transplant. 2019;34:90-9.

13. Macdougall IC, Akizawa T, Berns JS, Bernhardt T, Krueger T. Effects of molidustat in the treatment of anemia in CKD. Clin J Am Soc Nephrol. 2019; 14:28-39.

14. Besarab A, Provenzano R, Hertel J, Zabaneh R, Klaus SJ, Lee T, et al. Randomized placebo-controlled dose-ranging and pharmacodynamics study of roxadustat (FG-4592) to treat anemia in non-dialysis dependent chronic kidney disease (NDD-CKD) patients. Nephrol Dial Transplant. 2015; 30:1665-73.

15. Provenzano R, Besarab A, Sun CH, Diamond SA, Durham JH, Cangiano JL, et al. Oral hypoxia-inducible factor prolyl hydroxylase inhibitor roxadustat (FG-4592) for the treatment of anemia in patients with CKD. Clin J Am Soc Nephrol. 2016;11:982-91.

16. Holdstock L, Cizman B, Meadowcroft AM, Biswas N, Johnson BM, Jones D, et al. Daprodustat for anemia: a 24-week, open-label, randomized controlled trial in participants with chronic kidney disease. Clin Kidney J. 2019;12:129-38.

17. Pergola PE, Spinowitz BS, Hartman CS, Maroni BJ, Haase VH. Vadadustat, a novel oral HIF stabilizer, provides effective anemia treatment in nondialysisdependent chronic kidney disease. Kidney Int. 2016;90:1115-22.

18. Martin ER, Smith MT, Maroni BJ, Zuraw QC, DeGoma EM. Clinical trial of vadadustat in patients with anemia secondary to stage 3 or 4 chronic kidney disease. Am J Nephrol. 2017:45:380-8.

19. Akizawa T, Nangaku M, Yamaguchi T, Arai M, Koretomo R, Matsui A, et al. A placebo-controlled, randomized trial of enarodustat in patients with chronic kidney disease followed by long-term trial. Am J Nephrol. 2019;49:165-74.

20. Sender R, Fuchs $S$, Milo R. Revised estimates for the number of human and bacteria cells in the body. PLoS Biol. 2016;14(8):e1002533. https://doi.org/10. 1371/journal.pbio.1002533 eCollection 2016 Aug.

21. Yamamoto H, Nishi S, Tomo T, Masakane I, Saito K, Nangaku M, et al. 2015 Japanese Society for Dialysis Therapy: guidelines for renal anemia in chronic kidney disease. Ren Rep Ther. 2017;3(36):8-11.

22. Brannon ES, Merrill AJ, Warren JV, Stead EA Jr. The cardiac output in patients with chronic anemia as measured by the technique of right atrial catheterization. J Clin Invest. 1945;24(3):332-6.

23. Tanaka T, Eckardt KU. HIF activation against CVD in CKD: novel treatment opportunities. Semin Nephrol. 2018;38(3):267-76.

24. Silverberg DS, Wexler D, laina A. The importance of anemia and its correction in the management of severe congestive heart failure. Eur J Heart Fail. 2002:4(6):681-6.

25. Ayus JC, Go AS, Valderrabano F, Verde E, de Vinuesa SG, Achinger SG, et al. Spanish Group for the study of the anemia and left ventricular hypertrophy in pre-dialysis patients. Effects of erythropoietin on left ventricular hypertrophy in adults with severe chronic renal failure and hemoglobin < $10 \mathrm{~g} / \mathrm{dL}$. Kidney Int. 2005;68(2):788-95.

26. Akaishi M, Hiroe M, Hada Y, Suzuki M, Tsubakihara Y, Akizawa T, KRN321 Study Group. Effect of anemia correction on left ventricular hypertrophy in patients with modestly high hemoglobin level and chronic kidney disease. Cardiol. 2013;62(4):249-56. https://doi.org/10.1016/j.jjcc.2013.04.008 Epub 2013 Jun 18.

27. Kuriyama S, Tomonari H, Yoshida H, Hashimoto T, Kawaguchi Y, Sakai O. Reversal of anemia by erythropoietin therapy retards the progression of chronic renal failure, especially in nondiabetic patients. Nephron. 1997;77: $176-85$

28. Gouva C, Nikolopoulos P, loannidis JP, Siamopoulos KC. Treating anemia early in renal failure patients slows the decline of renal function: a randomized controlled trial. Gouva C, Nikolopoulos P, loannidis JP, Siamopoulos KC. Kidney Int. 2004;66(2):753-60.

29. Tsubakihara Y, Akizawa T, Iwasaki M, Shimazaki R. High hemoglobin levels maintained by an erythropoiesis-stimulating agent improve renal survival in patients with severe renal impairment. Ther Apher Dial. 2015;19(5):457-65. https://doi.org/10.1111/1744-9987.12308 Epub 2015 May 5.

30. Akizawa T, Tsubakihara $Y$, Hirakata $H$, Watanabe $Y$, Hase $H$, Nishi $S$, et al. A prospective observational study of early intervention with erythropoietin therapy and renal survival in non-dialysis chronic kidney disease patients with anemia: JET-STREAM Study. Clin Exp Nephrol. 2016;20:885-95

31. Hayashi T, Maruyama S, Nangaku M, Narita I, Hirakata H, Tanabe K, et al. Darbepoetin alfa in patients with advanced CKD without diabetes: randomized, controlled trial. Clin J Am Soc Nephrol. 2020. https://doi.org/10. 2215/CJN.08900719 [Epub ahead of print].

32. Besarab A, Bolton WK, Browne JK, Egrie JC, Nissenson AR, Okamoto DM, et al. The effects of normal as compared with low hematocrit values in patients with cardiac disease who are receiving hemodialysis and epoetin. N Engl J Med. 1998;339(9):584-90.

33. Drüeke TB, Locatelli F, Clyne N, Eckardt KU, Macdougall IC, Tsakiris D, et al. Normalization of hemoglobin level in patients with chronic kidney disease and anemia. N Engl J Med. 2006;355(20):2071-84.

34. Singh AK, Szczech L, Tang KL, Barnhart H, Sapp S, Wolfson M, et al. Correction of anemia with epoetin alfa in chronic kidney disease. N Engl J Med. 2006;355(20):2085-98.

35. Pfeffer MA, Burdmann EA, Chen CY, Cooper ME, de Zeeuw D, Eckardt KU, et al. A trial of darbepoetin alfa in type 2 diabetes and chronic kidney disease. N Engl J Med. 2009;361(21):2019-32. https://doi.org/10.1056/ NEJMoa0907845 Epub 2009 Oct 30

36. Swedberg K, Young JB, Anand IS, Cheng S, Desai AS, Diaz R, et al. Treatment of anemia with darbepoetin alfa in systolic heart failure. N Engl J Med. 2013; 368:1210-9.

37. Kuragano T, Kitamura K, Matsumura O, Matsuda A, Hara T, Kiyomoto H, et al. ESA hyporesponsiveness is associated with adverse events in maintenance hemodialysis patients, but not with iron storage. PloS One. 2016;11: e0147328.

38. McCullough PA, Barnhart HX, Inrig JK, Reddan D, Sapp S, Patel UD, et al. Cardiovascular toxicity of epoetin-alfa in patients with chronic kidney disease. Am J Nephrol. 2013;37:549-58.

39. Kanbay M, Perazella MA, Kasapoglu B, Koroglu M, Covic A. Erythropoiesis stimulatory agent-resistant anemia in dialysis patients: review of causes and management. Blood Purif. 2010;29:1-12. https://doi.org/10.1159/000245041.

40. Kidney Disease: Improving Global Outcomes (KDIGO) Anemia Working Group. KDIGO Practice Guideline for anemia in chronic kidney disease. Kidney Int. 2012;suppl 2:279-335.

41. Yamamoto H, Nishi S, Tomo T, Masakane I, Saito K, Nangaku M, et al. 2015 Japanese Society for Dialysis Therapy: guidelines for renal anemia in chronic kidney disease. Ren Rep Ther. 2017;3(36):11-7.

42. McFarlane SI, Chen SC, Whaley-Connell AT, Sowers JR, Vassalotti JA, Salifu $\mathrm{MO}$, et al. Prevalence and associations of anemia of CKD: Kidney Early Evaluation Program (KEEP) and National Health and Nutrition Examination Survey (NHANES) 1999-2004. Am J Kidney Dis. 2008;51(4 Suppl 2):S46-55. https://doi.org/10.1053/j.ajkd.2007.12.019.

43. Go AS, Chertow GM, Fan D, McCulloch CE, Hsu CY. Chronic kidney disease and the risks of death, cardiovascular events, and hospitalization. N Engl J Med. 2004;351(13):1296-305.

44. Himmelfarb J, Stenvinkel P, Ikizler TA, Hakim RM. The elephant in uremia: oxidant stress as a unifying concept of cardiovascular disease in uremia. Kidney Int. 2002;62:1524-38.

45. Otaki Y, Nakanishi N, Hasuike $Y$, Moriguchi M, Nanami $Y$, Hama M, et al. Defective regulation of iron transporters leading to iron excess in the polymorphonuclear leukocytes of patients on maintenance hemodialysis. Am J Kidney Dis. 2004;43:1030-9.

46. Nakanishi T, Kuragano T, Nanami M, Nagasawa Y, Nanami M, Hasuike Y. Misdistribution of iron and oxidative stress in chronic kidney disease. Free Rad Biol Med. 2019;133:248-53.

47. Honda H, Kobayashi Y, Onuma S, Shibagaki K, Yuza T, Hirao K, et al. Associations among erythroferrone and biomarkers of erythropoiesis and iron metabolism, and treatment with long-term erythropoiesis-stimulating agents in patients on hemodialysis. PloS One. 2016;11(3):e0151601. https:// doi.org/10.1371/journal.pone.0151601. 
48. Honda H, Hosaka N, Ganz T, Shibata T. Iron metabolism in chronic kidney disease patients. Nakanishi T, Kuragano T (eds): CKD-Associated Complications: Progress in the Last Half Century. Contrib Nephrol. 2019;198:103-11. https://doi.org/10.1159/000496369.

49. Peyssonnaux C, Zinkernagel AS, Schuepbach RA, Rankin E, Vaulont S, Haase $\mathrm{VH}$, et al. Regulation of iron homeostasis by the hypoxia inducible transcription factors (HIFs). J Clin Invest. 2007;117(7):1926-32. https://doi.org/ 10.1172/JCl31370.

50. Hasuike $Y$, Nonoguchi H, Tokuyama M, Ohue M, Nagai T, Yahiro M, et al. Serum ferritin predicts prognosis in hemodialysis patients: the Nishinomiya study. Clin Exp Nephrol. 2010;14(4):349-55. https://doi.org/10.1007/s10157010-0288-x Epub 2010 May 14.

51. Karaboyas A, Morgenstern H, Pisoni RL, Raymond Vanholder JZ, Jacobson SH, Inaba M, et al. Association between serum ferritin and mortality: findings from the USA, Japan and European Dialysis Outcomes and Practice Patterns Study. Nephrol Dial Transplant. 2018;33: 2234-44.

52. Hamano T, Fujii N, Hayashi T, Yamamoto H, Iseki K, Tsubakihara Y. Thresholds of iron markers for iron deficiency erythropoiesis-finding of the Japanese nationwide dialysis registry. Kidney Int Suppl. 2015;5(1):23-32. https://doi.org/10.1038/kisup.2015.6.

53. Shoji T, Niihata K, Fukuma S, Fukuhara S, Akizawa T, Inaba M. Both low and high serum ferritin levels predict mortality risk in hemodialysis patients without inflammation. Clin Exp Nephrol. 2017;21(4):685-93. https://doi.org/ 10.1007/s10157-016-1317-1.

54. Robinson BM, Bieber B, Pisoni RL, Port FK. Dialysis Outcomes and Practice Patterns Study (DOPPS): its strengths, limitations, and role in informing practices and policies. Clin J Am Soc Nephrol. 2012;7(11):1897-905. https:// doi.org/10.2215/CJN.04940512 Epub 2012 Oct 25.

55. Maruyama Y, Yokoyama K, Yokoo T, Shigematsu T, Iseki K, Tsubakihara Y. The different association between serum ferritin and mortality in hemodialysis ans peritoneal dialysis patients using Japanese nationwide dialysis survey. PLoS One. 10(11):e0143430. https://doi.org/10.1371/journal. pone.0143430.

56. Mursu J, Robien K, Harnack L, Park K, Jacobs DR Jr. Dietary supplements and mortality rate in older women: the lowa Women's Health Study. Arch Intern Med. 2011;171(18):1625-33. https://doi.org/10.1001/archinternmed. 2011.445.

57. Rostoker G, Griuncelli M, Loridon C, Magna T, Machado G, Drahi G, et al. Reassessment of iron marker for prediction of dialysis iron overload: an MRI study. PLoS One. 2015;10(7):e0132006. Published online 2015 Jul 16. https:// doi.org/10.1371/journal.pone.0132006.

58. Ogawa C, Tsuchiya K, Tomosugi N, Kanda F, Maeda K, Maeda T. Low levels of serum ferritin and moderate transferrin saturation leads to adequate hemoglobin levels in hemodialysis patients, retrospective observational study. PLos ONE. 2017;12(6):e0179608 June 29.

59. Anraku M, Kitamura K, Shintomo R, Takeuchi K, Ikeda H, Nagano J, et al. Effect of intravenous iron administration frequency on AOPP and inflammatory biomarkers in chronic hemodialysis patients: a pilot study. Clin Biochem. 2008;41(14-15):1168-74. https://doi.org/10.1016/j.clinbiochem. 2008.07.007 Epub 2008 Jul 28.

60. Yamamoto H, Nishi S, Tomo T, Masakane I, Saito K, Nangaku M, et al. 2015 Japanese Society for Dialysis Therapy: guidelines for renal anemia in chronic kidney disease. Ren Rep Ther. 2017;3(36):19-25.

61. Macdougall IC, White C, Anker SD, Bhandari S, Farrington K, Kalra PA, et al. Intravenous iron in patients undergoing maintenance hemodialysis. N Eng J Med. 2019;380:447-58.

62. Macdougall IC, Bhandari S, White C, Anker SD, Farrington K, Kalra PA, et al. Intravenous iron dosing and infection risk in hemodialysis patients: a prespecified secondary analysis of the PIVOTAL trial. J Am Soc Nephrol. 2020; 31:1118-27.

63. Coyne DW, Fishbane S. The value of intravenous iron: beyond the cave of speculation. J Am Soc Nephrol. 2020;31:896-7. https://doi.org/10.1681/ASN. 2019121340.

64. Kuo KL, Hung SC, Tseng WC, Tsai MT, Liu JS, Lin MH, et al. Association of anemia and iron parameters with mortality among patients undergoing prevalent hemodialysis in Taiwan: the AIM - HD study. J Am Heart Assoc. 2018;7(15):e009206.

65. Winkelmayer WC, Liu J, Brookhart MA. Altitude and all-cause mortality in incident dialysis patients. JAMA. 2009;301(5):508-12. https://doi.org/10.1001/ jama.2009.84.
66. Gupta N, Wish JB. Hypoxia-inducible factor prolyl hydroxylase inhibitors: a potential new treatment for anemia in patients with CKD. Am J Kidney Dis. 2017:69:815-26.

67. Yeh TZ, Leissing TM, Abboud MI, Thinnes CC, Atasoylu O, Holt-Martyn JP, et al. Molecular and cellular mechanisms of HIF prolyl hydroxylase inhibitors in clinical trials. Chem Sci. 2017;8:7651-68.

68. Tsubakihara Y, Akizawa T, Nangaku M, Onoue T, Yonekawa T, Matsushita H, et al. A 24-week anemia correction study of daprodustat in Japanese dialysis patients. Therapeutic Apheresis Dialysis. 2019. https://doi.org/10. 1111/1744-9987.12962

69. Akizawa T, Nangaku M, Yonekawa T, Okuda N, Kawamatsu S, Onoue T, et al. Efficacy and safety of daprodustat compared with darbepoetin alfa in Japanese hemodialysis patients with anemia: a randomized, double-blind, phase 3 trial. Clin J Am Soc Nephrol. 2020;15(8):1155-65. https://doi.org/10. 2215/CJN.16011219 Epub 2020 Jul 28.

70. Hara K, Takahashi N, Wakamatsu A, Caltabiano S. Pharmacokinetics, pharmacodynamics and safety of single, oral doses of GSK1278863, a novel HIF-prolyl hydroxylase inhibitor, in healthy Japanese and Caucasian subjects. Drug Metab Pharmacokinet. 2015;30(6):410-8. https://doi.org/10.1016/j. dmpk.2015.08.004 Epub 2015 Aug 28.

71. Akizawa T, Iwasaki M, Otsuka T, Reusch M, Misumi T. roxadustat treatment of chronic kidney disease-associated anemia in Japanese patients not on dialysis: a phase 2, randomized, double-blind, placebo-controlled trial. Adv Ther. 2019;36(6):1438-54. https://doi.org/10.1007/s12325-019-00943-4 Epub 2019 Apr 5.

72. Akizawa T, Ueno M, Shiga $T$, Reusch M. Oral roxadustat three times weekly in ESA-naïve and ESA-converted patients with anemia of chronic kidney disease on hemodialysis: results from two phase 3 studies. Ther Apher Dial. 2019. https://doi.org/10.1111/1744-9987. 13468 [Epub ahead of print].

73. Akizawa T, Otsuka T, Reusch M, Ueno M. Intermittent oral dosing of roxadustat in peritoneal dialysis chronic kidney disease patients with anemia: a randomized, phase 3, multicenter, open-label study. Ther Apher Dial 2020;24(2):115-125. doi: https://doi.org/10.1111/1744-9987.12888. Epub 2019 Jul 31.

74. Chen N, Hao C, Liu BC, Lin H, Wang C, Xing C, et al. Roxadustat treatment for anemia in patients undergoing long-term dialysis. $\mathrm{N}$ Engl J Med. 2019;381(11):1011-22. https://doi.org/10.1056/NEJMoa1901713 Epub 2019 Jul 24.

75. Akizawa T, Macdougall IC, Berns JS, Bernhardt T, Staedtler G, Taguchi M, et al. Long-term efficacy and safety of Molidustat for anemia in chronic kidney disease: DIALOGUE extension studies. Am J Nephrol. 2019;49:271-80.

76. Akizawa T, Macdougall IC, Berns JC, Yamamoto H, Taguchi M, lekushi K, et al. Ion regulation by Molidustat, a daily oral hypoxia inducible factor prolyl hydroxylase inhibitor, in patients with chronic kidney disease. Nephron. 2019;143:243-57.

77. Akizawa T, Hanaki K, Arai M. JTZ-951 an oral novel HIF-PHD inhibitor, elevates hemoglobin in Japanese anemic patients with chronic kidney disease receiving maintenance hemodiaysis. 52nd ERA-EDTA. London; 2015.

78. Zhong H, Zhou T, Li H, Zhong Z. The role of hypoxia-inducible factor stabilizers in the treatment of anemia in patients with chronic kidney disease. Drug Des Devel Ther. 2018;12:3003-11. https://doi.org/10.2147/ DDDT.S175887 eCollection 2018.

79. Haase VH. HIF-prolyl hydroxylases as therapeutic targets in erythropoiesis and iron metabolism. Hemodial Int. 2017;21(Suppl 1):S110-24. https://doi. org/10.1111/hdi.12567.

80. Roxadustat phase III programme pooled analyses showed positive efficacy and no increases cardiovascular risk in patients with anaemia from chronic kidney disease. http://www.astrazeneca.co./mediacentre/press-release/2019/ rozadustat-phase-iii-programme-pooled-analyse-showed-positive-efficacyand-no-increased-cv-risk-in-patients-with-anaemia-from-chronic-kidneydisease.html. (Accessed June 15, 2020).

81. Sanghani NS, Hasse VH. Hypoxia-inducible factor activators in renal anemia: current clinical experience. Adv Chronic Kidney Dis. 2019;26:253-66.

82. Flamme I, Oehme F, Ellinghaus P, Jeske M, Keldenich M, Thuss U. Mimicking hypoxia to treat anemia: HIF-stabilizer BAY 85-3934 (Molidustat) stimulates erythropoietin production without hypertensive effects. Plos One November. 2014;9(11):e111838.

83. Uchida K, Nangaku M, Abe M, Okada H, Takeda N, Hanafusa N, et al. Recommendation on the appropriate use of HIF-PH inhibitors: Japanese Society of Nephrology. 2020. (in Japanese). 
84. Sofue T, Nakagawa N, Kanda E, Nagasu H, Matsushita H, Nangaku M, et al. Prevalence of anemia in patients with chronic kidney disease in Japan: a nationwide, cross-sectional cohort study using data from the Japan Chronic Kidney Disease Database (JCKD-DB). Plos One. 2020;15(7):e0236132. https:// doi.org/10.1371/journal.pone.0236132.

\section{Publisher's Note}

Springer Nature remains neutral with regard to jurisdictional claims in published maps and institutional affiliations.

Ready to submit your research? Choose BMC and benefit from:

- fast, convenient online submission

- thorough peer review by experienced researchers in your field

- rapid publication on acceptance

- support for research data, including large and complex data types

- gold Open Access which fosters wider collaboration and increased citations

- maximum visibility for your research: over $100 \mathrm{M}$ website views per year

At $\mathrm{BMC}$, research is always in progress.

Learn more biomedcentral.com/submissions 